\title{
COMPACTIFICATIONS OF SEMITOPOLOGICAL SEMIGROUPS II
}

\author{
PAUL MILNES ${ }^{1}$
}

(Received 7 November, 1973)

\begin{abstract}
Correcting some "proofs" given in an earlier paper of the same title, we prove here, among other things, that, if $S$ is a subgroup of a topological group that is complete in a left invariant metric or locally compact, then every weakly almost periodic function on $S$ is (left and right) uniformly continuous. We also prove a theorem related to results of $\mathbf{R}$. B. Burckel and of W. W. Comfort and K. A. Ross: a topological group is pseudocompact if and only if $\operatorname{WAP}(G)=C(G)$.
\end{abstract}

It has been pointed out to us that there is a gap in the proof of Theorem 4.6 of Milnes (1973). Thus the proofs of Corollary 4.7 and Theorem 4.8, which refer to Theorem 4.6, appear to have gaps as well. We prove here that the assertions of Theorem 4.6 and Corollary 4.7 are true at least if an additional assumption is made and that that of Theorem 4.8 as stated in Milnes (1973) is true. We also discuss examples which show that some of the hypotheses of these results are necessary. We do not know of an example which shows that the statement of Theorem 4.6 in Milnes (1973) is false, and conjecture that this statement is in fact true. In conclusion, we prove a theorem related to results in Burckel (1970), Comfort \& Ross (1966), Milnes (1973): a topological group $G$ is pseudocompact if and only if WAP $(G)=C(G)$.

Suppose $S$ is a semitopological semigroup and $\beta S$ is the spectrum of $C(S)$. A closed linear subspace $X$ of $C(S)$ is called left $m$-introverted if the function $s \rightarrow x\left(f_{s}\right)$ is in $X$ whenever $f \in X$ and $x$ is a continuous multiplicative linear functional on $C(S)$. (Here $f_{s}$ is the left translate of $f$ by $s$, which is defined by $f_{s}(t)=f(s t) \forall t \in S$.) Such subspaces were introduced by Mitchell (1970), who

'This research was supported in part by a National Research Council grant. 
showed that there exists a greatest such subspace $\operatorname{LMC}(S)$, which may be characterized by

$$
\operatorname{LMC}(S)=\left\{f \in C(S) \mid \text { the function } s \rightarrow x\left(f_{s}\right) \text { is in } C(S) \forall x \in \beta S\right\} .
$$

In case $S$ is a topological group $G$ that is locally compact or complete in a left invariant metric, Mitchell (see Rao (1965) as well) also proved that $\operatorname{LMC}(G)=$ LUC $(G)$, the left uniformly continuous subspace of $C(G)$. It is our purpose here to prove the following results.

THEOREM 1. Suppose $S$ is a dense subgroup of a compact topological group $G$. Then every member of $L M C(S)$ extends (uniquely by continuity) to a member of $L M C(G)$, i.e., $\left.L M C(G)\right|_{s}=L M C(S)$.

Corollary 2. Suppose $S$ is (a topological group homeomorphic and isomorphic to) a subgroup of a compact topological group $G$. Then $L M C(S)=$ $\operatorname{LUC}(S)$.

In Milnes (1973) we thought we had proved these results without the assumption that $G$ is compact. However, it has been pointed out to us that there is a gap in the proof given there. Nonetheless, we do not know that this asssumption is necessary and make the

Conjecture. The conclusions of Theorem 1 and Corollary 2 still hold if the hypothesis that $G$ is compact is dropped.

Proof of Theorem 1. Since it is clear that $\left.\mathrm{LMC}(G)\right|_{s} \subset \mathrm{LMC}(S)$, we need only show the reverse inclusion. Let $f \in \operatorname{LMC}(S)$. By Lemma 4.5 of Milnes (1973) $f$ extends (uniquely by continuity) to a function in $C(G)$. Since $G$ is compact, $C(G)=\mathrm{LMC}(G)$ and we are done.

Proof of Corollary 2. By Theorem 1, LMC $\left.(G)\right|_{s}=\mathrm{LMC}(S)$. But $\operatorname{LMC}(G)=\operatorname{LUC}(G)$ and the restriction of a function in $\operatorname{LUC}(G)$ to $S$ is in $\operatorname{LUC}(S)$.

Remark. It follows from Theorem 1 and a result of Weil (1937) that $\mathrm{LMC}(S)=\mathrm{LUC}(S)=\mathrm{WAP}(S)=\operatorname{AP}(S)$ whenever $S$ is a totally bounded topological group, exactly as in the case where $S$ is compact. (When $S$ is compact, $C(S)=\mathrm{AP}(S)$ as well; see also $\$ 3$ ahead.)

Some examples are now presented, where the conclusion of Theorem 1 fails. The first is due to T. Mitchell; the third was communicated to us by J. F. Berglund.

EXAMPLE 1 [Granirer and Lau (1971); p. 257]. Consider the subsemigroup $S=(0, \infty)$ of the usual additive reals $\mathrm{R}$. Then the function $t \rightarrow \sin (1 / t)$ is in LUC $(S) \subset \mathrm{LMC}(S)$, but does not extend to a function continuous on $R$, or, for 
that matter, to a function continuous on the subsemigroup $[0, \infty)$ of $R$ in which $S$ is dense.

EXAMPLE 2. Let $T$ be the one point compactification of the subsemigroup $S=[1, \infty)$ of the usual additive reals and suppose multiplication is extended from $S$ to $T$ by making the adjoined point a zero for $T$. Then the only functions in $C(S)$ that extend to functions in $C(T)$ are those that tend to a limit at infinity. Thus the function $t \rightarrow \sin t$ is in $\operatorname{LUC}(S) \subset \operatorname{LMC}(S)$, but has no continuous extension to $T$.

Example 3. Let $R$ be the usual additive reals and adjoin a zero $\theta$ at $+\infty$ to get a (non-compact) topological semigroup $T=R \cup\{\theta\}$ where, for a net $\left\{t_{\alpha}\right\} \subset R, t_{\alpha} \rightarrow \theta$ is equivalent to $t_{\alpha} \rightarrow+\infty$. Then the function $t \rightarrow \tan ^{-1} t$ is in $\operatorname{LUC}(R)=\operatorname{LMC}(R)$ and has an extension which is continuous on $T$. However, this extension is not in $\operatorname{LMC}(T)$ : the sequence of translates of this extension by the members $\{-n\}_{n=1}^{x} \subset R$ converges pointwise on $T$ to a function $f$ such that $f(t)=-\pi / 2$ for $t \in R, f(\theta)=\pi / 2$. Thus $f \notin C(T)$ and the extension is not in $\mathrm{LMC}(T)$ [Milnes (1973); Theorem 3.1].

2

If $G$ is a topological group, we write $\operatorname{WAP}(G)$ for the weakly almost periodic subspace of $C(G)$ and note that both methods of proof given in Milnes (1973) of the theorem which asserts that WAP $\left.(G)\right|_{s}=\operatorname{WAP}(S)$ whenever $S$ is a dense subgroup of a topological group $G$ [Milnes (1973); Theorem 4.9 (i)] are correct; in particular, the method which refers to the proof of Theorem 4.6 is correct.

Also in Milnes (1973) we gave an example of a locally compact group $G$ with closed normal abelian subgroup $H$ such that no non-trivial character of $H$ extends to a function (left and right) uniformly continuous on $G$. Hence WAP $\left.(G)\right|_{H}$ does not even contain $\operatorname{AP}(H)$. In view of this "bad behaviour", it seems strange that $\left.\operatorname{LMC}(G)\right|_{H}=\mathrm{LMC}(H)$ (here and whenever $H$ is a closed subgroup of a topological group that is locally compact or complete in a left invariant metric). This follows from theorems of Mitchell mentioned above and a theorem of Katětov $(1951,1953)$.

Next, the result mentioned at the beginning of this section is used to prove

Theorem 3 [Milnes (973); Theorem 4.8]. If $S$ is (homeomorphic and isomorphic to) a subgroup of a topological group that is complete in a left invariant metric or locally compact, then all functions in WAP(S) are (left and right) uniformly continuous.

Proof. By Milnes (1973; Theorem 4.9 (i)), WAP $\left.(G)\right|_{s}=$ WAP(S). Also, 
WAP $(G) \subset \operatorname{LMC}(G)=\operatorname{LUC}(G)$ in this setting [Mitchell (1970)], and the map $f \rightarrow \check{f}$, where $\check{f}(s)=f\left(s^{-1}\right) \forall s \in G$, maps $\operatorname{WAP}(G)$ onto $\operatorname{WAP}(G)$ and $\operatorname{LUC}(G)$ onto RUC(G), the right uniformly continuous subspace of $C(G)$. It follows that WAP $(G)$ consists of (left and right) uniformly continuous functions and hence that $\operatorname{WAP}(S)=\left.\operatorname{WAP}(G)\right|_{s}$ does too.

In Burckel $(1970 ; 2.24$, p. 31 and Theorem 4.10,p. 68), it is proved that, for a locally compact topological group $G$, each of the equalities, $\operatorname{AP}(G)=\operatorname{WAP}(G)$ and WAP $(G)=C(G)$, implies that $G$ is compact. Subsequently, it has been shown that the local compactness hypothesis is necessary in both cases. In Milnes (1973; Corollary 4.10) we showed that $\operatorname{AP}(G)=\mathrm{WAP}(G)$ whenever $G$ is a totally bounded topological group (for example, if $G$ is the group of rational numbers (modulo 1)). And Burckel (1970) has constructed a topological group $G$ which is not locally compact and for which$$
\operatorname{AP}(G)=\operatorname{WAP}(G)=C(G) .
$$

It follows from a theorem of Comfort and Ross (1966) that this group is pseudocompact and that $*$ holds for all pseudocompact groups $G$. This raises the question: is there a topological group $G$ for which WAP $(G)=C(G)$, but $\operatorname{AP}(G) \neq \operatorname{WAP}(G)$ ? That the answer to this question is no is a consequence of the following

THEOREM 4. Let $G$ be a topological group for which $W A P(G)=C(G)$. Then $A P(G)=C(G)$ as well and hence $G$ is pseudocompact.

Proof. As a first step, we invoke Burckel (1970; Theorem 4.10, p. 68) and may assume without loss that $G$ is not discrete. We next prove that $G$ is totally bounded. The proof is by contradiction. Suppose $G$ is not totally bounded. Then $\exists$ a neighbourhood $V$ of $e \in G$ and a sequence $\left\{s_{n}\right\}_{n-1}^{\infty} \subset G$ such that

$$
\text { ** } \quad V s_{n} \cap V s_{m}=\varnothing \quad \text { if } \quad m \neq n \text {. }
$$

By induction we can get a sequence $\left\{f_{n}\right\} \subset C(G)$, a sequence $\left\{V_{n}\right\}$ of neighborhoods of $e \in G$ and a sequence $\left\{t_{n}\right\} \subset G$ such that:

(i) $V_{1} \subset V, V_{n+1} \subset V_{n}$

(ii) $f_{n}=0$ off $V_{n}, f_{n}=1$ on $V_{n+1}, 0 \leqq f_{n} \leqq 1$.

(iii) $t_{n} \in V_{n} \backslash V_{n+1}$.

Now $\forall n$ let $h_{n} \in C(G)$ be defined by

$$
h_{n}(t)=f_{n}\left(t s_{n}^{-1}\right) \quad \text { and put } \quad h=\sum h_{n} .
$$

It follows from ${ }^{* *}$ that $h \in C(G)$. Also, $h \notin \mathrm{WAP}(G)$, which is the desired contradiction. For 


$$
h\left(t_{m} s_{n}\right)=f_{n}\left(t_{m}\right)=\left\{\begin{array}{lll}
1 & \text { if } & m>n \\
0 & \text { if } & m<n
\end{array} \quad\right. \text { and hence }
$$

$$
\lim _{m} \lim _{n} h\left(t_{m} s_{n}\right)=0 \text { and } \lim _{n} \lim _{m} h\left(t_{m} s_{n}\right)=1
$$

which is impossible for a function in WAP $(G)$ [Grothendieck (1952)].

Thus $G$ is totally bounded and, by Corollary 4.10 of Milnes (1973), $\operatorname{AP}(G)=\mathrm{WAP}(G)$. Hence $\operatorname{AP}(G)=C(G)$; and $G$ is pseudocompact by the result of Comfort and Ross already mentioned.

REMARKs. (a) It is easy to see that one can have $\mathrm{AP}(G)=\mathrm{WAP}(G)$ and WAP $(G) \neq C(G)$ for the same topological group $G$. For example, let $G$ be any non-pseudocompact totally bounded topological group (such as the rational numbers (modulo 1)).

(b) Theorem 4.10 of Burckel (1970) may be construed as an easy corollary of the proof of Theorem 4 above. This is because a locally compact totally bounded topological group must in fact be compact.

(c) If $S$ is a topological semigroup, one would not expect the equality WAP $(S)=C(S)$ always to imply $\operatorname{AP}(S)=C(S)$, and indeed this is not the case. Macri (1974) has provided the following

Example. Let $S$ be an infinite set with distinct elements $1,0 \in S$. The multiplication rules

$$
\begin{array}{lll}
a b=0 & \text { if } & a \neq b \\
a^{2}=1 \quad \text { for } & a \neq 0,1 \\
0^{2}=1^{2}=0 &
\end{array}
$$

make $S$ a (discrete) semigroup. To see that WAP $(S)=C(S)$ let $f \in C(S)$. We assume without loss that $f(0)=0$ and must show that, if $\left\{a_{n}\right\} \subset S$, then a subsequence of $\left\{f_{a_{n}}\right\}$ converges weakly in $C(S)$. To this end, we may assume either

(i) $a_{n}=a$, a fixed.member of $S \forall n$, or (ii) $a_{n} \neq a_{m}$ if $n \neq m$.

In case (i) the sequence $\left\{f_{a_{n}}\right\}$ is constant and in case (ii) each $f_{a_{n}}$ is 0 except, possibly, at $a_{n}$; thus $f_{a_{n}} \rightarrow 0$ uniformly on compacta (which are finite sets here), and in bounded subsets of $C_{0}(S)$ uniform convergence on compacta implies weak convergence.

A function $f \in C(S), f \notin \mathrm{AP}(S)$, can be defined by

$$
f(a)= \begin{cases}0 & \text { if } a=0 \\ 1 & \text { otherwise }\end{cases}
$$


For, if $\left\{a_{n}\right\} \subset S \backslash\{0,1\}$ satisfies $a_{n} \neq a_{m}$ if $n \neq m$, then $\forall n$

$$
f_{a_{n}}(a)=\left\{\begin{array}{cc}
1 & \text { if } a=a_{n} \\
0 & \text { otherwise. }
\end{array}\right.
$$

Hence $f_{a_{n}} \rightarrow 0$ uniformly on compacta, but not uniformly: $f \notin \operatorname{AP}(S)$.

Note added in proof. The conjecture mentioned in the first paragraph and stated after Corollary 2 is false; see Counter-example in the theory of continuous functions on topological groups, by P. Milnes and J. S. Pym (to appear in Pacific J. Math.).

\section{References}

R. B. Burckel (1970), 'Weakly Almost Periodic Functions on Semigroups', Gordon and Breach, New York.

R. B. Burckel, 'Mimeographed addendum to Weakly Almost Periodic Functions on Semigroups'.

W. W. Comfort and K. A. Ross, (1966), 'Pseudocompactness and uniform continuity in topological groups', Pacific J. Math. 16, 483-496.

E. Granirer and A. T. Lau (1971), 'Invariant means on locally compact groups', Illinois J. Math. 15, 249-257.

A. Grothendieck (1952), 'Critères de compacité dans les espaces fonctionnels généraux', Amer. J. Math. 74, 168-186.

M. Katětov (1951, 1953), 'On real-valued functions in topological spaces', Fund. Math. 38, 85-91, and correction Fund. Math. 40, 203-205.

N. Macri (1974), 'The continuity of Arens product on the Stone-Cech compactification of semigroups', Trans. Amer. Math. Soc. 191, 185-193.

P. Milnes (1973), 'Compactifications of semitopological semigroups', J. Austral. Math. Soc. 15, 488-503.

T. Mitchell (1970), 'Topological semigroups and fixed points', Illinois J. Math. 14, 630-641.

C. R. Rao (1965), 'Invariant means on spaces of continuous or measurable functions', Trans. Amer. Math. Soc. 114, 187-196.

A. Weil (1937), 'Sur les espaces à structure uniforme et sur la topologie générale', Hermann, Paris.

Department of Mathematics

University of Western Ontario

London 72

Canada. 